Estudios sobre el Mensaje Periodístico

ISSN-e: $1988-2696$

http://dx.doi.org/10.5209/ESMP.55620

\title{
Mediación familiar en el uso de servicios digitales por menores escolarizados
}

\author{
Teresa Torrecillas-Lacaveㄹ ${ }^{1}$ María Encina Morales de Vega²; Tamara Vázquez-Barrio³
}

Recibido: 3 de mayo de 2016 / Aceptado: 28 de octubre de 2016

Resumen. Los recientes estudios de la recepción prestan especial atención a las influencias que ejercen distintos agentes de mediación en las relaciones que los menores establecen con los medios. El objetivo de este artículo es identificar estilos de mediación parental en el uso de Internet en el hogar por parte de menores escolarizados de la Comunidad de Madrid. Mediante encuesta hecha a padres de menores de entre 11 y 15 años, se ha procedido a hacer un análisis factorial con el que se han simplificado variables y un análisis de conglomerados del que se ha extraído una tipología compuesta por familias preocupadasausentes, preocupadas orientadoras, despreocupadas permisivas y despreocupadas controladoras. Esta investigación pone de manifiesto la necesidad de potenciar la educomunicación de los padres para que puedan ejercer una mediación adecuada.

Palabras clave: Nuevas tecnologías; internet; mediación parental; alfabetización mediática; medios de comunicación.

\section{[en] Family mediation in the use of digital services by children in schools}

Abstract. Recent studies of receiving pay special attention to the influences exerted by various actors in mediating the relationships that children establish with the media. The aim of this article is to identify styles of parental mediation in the use of Internet at home by the children. From a survey of parents of children between 11 and 15 years in the region of Madrid, it has been applied, first, a factorial analysis in order to simplify variables, and second, a cluster analysis to obtain a household typology composed by concerned - absent families, concerned guiding, permissive carefree and carefree controlling. This research exposes the need to promote media education of parents in order to exercise an adequate mediation.

Keywords: New technologies; internet; parental mediation; media literacy; media.

Sumario. 1. Introducción y estado de la cuestión. 2. Material y métodos. 3. Tipología de estilos de mediación; 3.1. Análisis y resultados. 4. Discusión y conclusiones. 5. Referencias bibliográficas.

Cómo citar: Torrecillas-Lacave, Teresa; Morales de Vega, María Encina; y Vázquez-Barrio, Tamara (2017): "Mediación familiar en el uso de servicios digitales por menores escolarizados", en Estudios sobre el Mensaje Periodístico 23 (1), 663-673.

1 Universidad CEU San Pablo.

E-mail: teresat@ceu.es

2 Universidad CEU San Pablo.

E-mail: memorales@ceu.es

3 Universidad CEU San Pablo.

E-mail: tamarav@ceu.es 


\section{Introducción y estado de la cuestión}

El avance de la Sociedad de la Información en España es imparable. 2014 ha sido el año de la consolidación de un nuevo paradigma mediático caracterizado por nuevos hábitos de producción y consumo de contenidos multimedia (Informe Telefónica, 2014). Este nuevo ambiente mediático se caracteriza por el hecho de que cualquier persona pueda erigirse en prosumidor (Sandoval y Aguaded, 2012), es decir, dotado de la posibilidad de crear y difundir contenidos de cualquier naturaleza además de acceder a ellos; por la ubicuidad, entendida como la posibilidad de poder disfrutar en cualquier momento y lugar de servicios de información (Islas Carmona, 2008: 35); por el consumo multidispositivo, según las circunstancias de acceso y el tipo de contenido a consumir; por el acceso en movilidad y por ser una comunicación intensiva social que gira alrededor de la idea de comunidad. Es un escenario en el que predomina, entre las generaciones más jóvenes, un perfil de consumidor digital permanente muy ligado a las redes sociales (Informe Telefónica, 2014).

En este contexto, la educomunicación es fundamental para la participación activa de los ciudadanos en la esfera pública, el ejercicio de derechos, la empleabilidad, la formación, el emprendimiento o la innovación (Aguaded, 2011: 7) y, en el caso de los menores, los agentes con responsabilidad en su educación - organismos políticos internacionales y nacionales, escuelas, familias y otros entes sociales - deben, por un lado, potenciar al máximo las oportunidades de la red y, por otro lado, reducir los riesgos porque el impacto de los contenidos de internet en los menores está condicionado por factores individuales y de contexto (Garitaonaindiay al., 2011: 77).

Desde la investigación en el ámbito académico, las líneas más actuales se centran en promover un uso seguro de Internet entre menores a partir de la educomunicación (Ferrés, Aguaded y García Matilla, 2012; Pérez Tornero y Varis, 2012; Pérez Tornero, 2015) como vía para el empoderamiento de una sociedad prosumidora que solo es posible mediante la alfabetización en competencias mediáticas (Marta Lazo y Grandío López, 2013).

En este sentido, este artículo pretende ser una aportación a los Estudios de Recepción en contextos familiares multipantallas y a la mediación familiar como uno de los retos más importantes que tiene frente a sí la investigación en educomunicación, por ser una de las vías más importantes para la inclusión digital de los menores.

El objetivo concreto de este artículo es, después de hacer un recorrido por los antecendentes, obtener una tipología de familias según sus estilos de mediación en el uso y consumo que hacen los menores escolarizados en el hogar de servicios digitales. Además se pretende incidir en la importancia de los padres en la educomunicación de los hijos.

La hipótesis que sustenta esta línea de investigación es que las distintas formas de recepción multipantalla de menores escolarizados está condicionada por el modo en el que los distintos agentes con responsabilidad en la protección de los menores (estado, colegio y padres) crean oportunidades educativas para una producción y consumo de contenidos digitales enriquecedora. La hipótesis que tratamos de verificar en este trabajo es que los estilos de mediación en contextos multipantalla están condicionados por la percepción que tengan los padres sobre la importancia de las TIC para el desarrollo y la socialización de los hijos y por el grado de conocimiento que tengan sobre los servicios y herramientas de Internet. 
La educación mediática se entiende desde una perspectiva holística que trasciende la mera capacitación para la utilización de las tecnologías para acceder a la información o producirla - dimensión que precisamente los menores tienen bien asimilada - y que abarca la asimilación de la dimensión social de su uso a la hora de comprender los sentidos y significaciones propuestos por los textos mediáticos y las repercusiones y responsabilidad de la creación y difusión de contenidos: lectura y creación de contenidos desde una perspectiva crítica y funcional (Gutiérrez y Tyner, 2012: 33). Como afirman Orozco, Navarro y García Matilla (2012: 70), el desafío consiste en convertir a las audiencias más o menos activas en usuarios en el momento en el que la interactividad trasciende la mera reacción a un estímulo y es fruto de una elaboración cognitiva, consciente y de decisión. La educación mediática persigue una formación integral (Aguaded, 2010: 63) que potencie la excelencia personal, la autonomía de los ciudadanos y el compromiso social y cultural que pasa por el fomento de una participación crítica en el ciberespacio (Kendall y McDougall, 2012: 21). La alfabetización digital es, por tanto, una necesidad de la ciudadanía democrática; se concibe como una derecho - tal y como queda reflejado en la Ley General Audiovisual 2011, en su artículo 6.4- necesario para el progreso y evitar desigualdades (Area, 2012: 28).

Tal y como hemos definido, los padres, al margen de todas las políticas que pueda implementar el estado para garantizar el derecho a la alfabetización mediática, o a la proyección de esta materia en los centros educativos, son los principales responsables de la inclusión digital de sus hijos porque el hogar es el lugar común de uso de Internet y porque los padres son el primer punto de contacto que los menores encuentran cuando tienen dificultades online (Livingstone y Bober, 2006).

Asumida la capacidad de interacción de los usuarios, y a la luz de las teorías ecológicas del desarrollo (Bronffenbrener, 1987), los estudios culturales británicos (Stuart Hall, 2004; Lull, 1997) o las teorías de la recepción (Orozco,1996), es necesario estudiar los procesos de codificación y decodificación de mensajes en sus contextos de recepción porque la apropiación de sentidos y significaciones está condicionada por aspectos socioculturales y por la acción de los distintos agentes de mediación que hacen de filtro.

Para comprender la naturaleza de las relaciones de los menores con internet hay que tener en cuenta las relaciones sociales que viven en su entorno más natural y cercano que es la familia (Morley: 1986). La mediación familiar, tomando las definiciones de Martín Barbero (1987) y Orozco (1996), la entendemos como instancia cultural desde donde los menores producen y se apropian de los sentidos y significaciones de los nuevos procesos comunicativos y que, por tanto, configura y reconfigura tanto la interacción con los nuevos servicios digitales — consumo y producción - como la creación del sentido de la interacción. Como afirman Livingstone y Helsper (2008), el concepto mediación "is widely seen to capture the parental management of the relation between children and media".

En los últimos años se ha mostrado especial atención a los riesgos a los que se exponen los menores pero poco sabemos de las estrategias de intervención socioeducativas de los padres. Un interesante trabajo que hace una revisión de la investigación sobre la interacción de niños y jóvenes con los nuevos medios, pone de manifiesto que frente a la obvia importancia de la mediación familiar, llama la atención que en los estudios se incluyen pocas variables familiares y que se profundice poco en los contextos de experiencias en Internet. Además, constata la dificultad de aportar solu- 
ciones adecuadas a la intervención de los adultos porque no hay evidencia empírica que pruebe cuáles son las estrategias de mediación realmente efectivas (Marciales y Cabra, 2011: 861-862).

En la literatura científica reciente se encuentra abundancia de trabajos sobre mediación parental en contextos televisivos (Valkenburgy al., 1999; van der Voorty al., 1992). Como afirman Livingstone y Helsper las estrategias se pueden clasificar en tres grandes grupos:

"Active mediation consist of talking about media content while the child is engaging with the mediun; restrictive mediation involves setting rules that restrict use of the médium; and co-using signifies that the parent remains present while the child is engaged with the medium" (Livingstone \& Helsper, 2006: 4).

En el ámbito español, un trabajo publicado fruto de investigaciones precedentes de este equipo, destaca la existencia de cuatro estilos de mediación condicionados por la posición de la familia en la estructura social: autocontrol, heterocontrol, control y descontrol. De todos éstos, el estilo de mediación predominante en los hogares es el de heterocontrol, "caracterizado por un alto grado de intervención en las relaciones de los hijos y el medio dado por normas parciales que surgen de momentos de control y covisión y que giran alrededor de los tiempos de consumo, los contenidos que consideran inadecuados por sexuales o violentos y los formatos como realities o programas del corazón".

En los últimos años emerge como una línea de investigación importante la mediación parental en contextos multipantallas con el objeto documentar cómo, porqué y con qué consecuencias los padres tratan de influir en la experiencia de los menores con las TIC (Haddon, 2015: 1). En contextos multipantallas hay muy poca acumulación de conocimiento. Entre los más recientes publicados en nuestro país destaca el desarrollado por Garmendia, y al. que destaca la amplia difusión de estrategias activas basadas en el diálogo y la orientación frente a las estrategias restrictivas basadas en normas (2013: 112). El trabajo de Pérez Sánchez constata que el uso intensivo de internet y videojuegos es predictivo del tipo de mediación permisiva, más presente en escuelas privadas y en niños de mayor edad, que además son más proclives a riesgos asociados al uso de redes sociales (2013). El trabajo de Aierbe, Orozco y Medrano, con muestra española y latinoamericana, pone de manifiesto que el estilo de mediación más percibido por los adolescentes es el co-viewing, seguido por el instructivemediation y finalmente por el restrictivemediation (93-94: 2014).

Hay autores que consideran que estrategias de control son perjudiciales porque cohíben la autonomía del niño (Stacksrud y Livingstone, 2009) frente a otros que apuestan por tutelar el uso y consumo con ayudas técnicas y normas (Bringué y Sádaba, 2008).

\section{Material y métodos}

Para cumplir el objetivo se utilizarán datos empíricos extraídos de una encuesta estadística representativa del conjunto de familias de la Comunidad de Madrid con hijos escolarizados. 
La encuesta se hizo a padres con menores de edades comprendidas entre los 11 y los 15 años, concretamente con hijos en $6^{\circ}$ de primaria, y $1^{\circ}$ y $2^{\circ}$ de secundaria. El ámbito de estudio se limitó a la Comunidad de Madrid. El procedimiento de muestreo fue probabilístico, aleatorio simple, estratificado (colegios públicos, privados y concertados), con afijación igual (400 unidades de análisis por cada unidad de muestreo).

El trabajo de campo se realizó entre febrero y junio de 2013. El cuestionario se distribuyó en una carta dirigida a los padres a través de los colegios. El error muestral resultante asociado a la encuesta fue del $2 \%$ para un nivel de confianza del $95 \%$.

Las técnicas estadísticas multivariables utilizadas para conseguir los diferentes estilos de mediación familiar han sido dos. En primer lugar, se ha realizado un análisis factorial con el objetivo de reducir la variedad de respuestas obtenidas en el cuestionario, de forma que se consiguiera un agrupamiento en base a rasgos similares. Una vez simplificadas en variables análogas, se ha realizado un análisis cluster para identificar los diferentes modelos de mediación familiar en cuanto a uso y consumo de TIC en los hogares.

El procedimiento de análisis y tratamiento de datos se ha llevado a cabo con el paquete estadístico IBM SPSS Satatics 19.0.

Este trabajo presenta un avance en el estudio de la mediación familiar por indagar en los contextos de experiencia en el uso y consumo de servicios digitales en el hogar por menores incluyendo, además de variables sobre estrategias de control, habituales en trabajos precedentes, como el tiempo, normas, límites, covisión, orientación o diálogo, otras variables relativas a la percepción de los padres sobre el papel de los servicios digitales en el desarrollo de los hijos y sobre el conocimiento que tienen del uso y consumo real que hacen. Se han utilizado un total de 45 preguntas del cuestionario.

\section{Tipología de estilos de mediación}

Se ha aplicado el análisis factorial sobre 45 preguntas realizadas, diferenciando entre el nivel multirrespuesta de cada pregunta, con el objetivo de obtener resultados metodológicamente correctos. El método de extracción ha sido el análisis de componentes principales, así como la rotación se ha realizado con normalización Varmimax con Kaiser.

En este sentido, el tratamiento de las preguntas con tres posibles respuestas, ha permitido reducir un total de 16 variables a 5 factores que explican el $60 \%$ de la varianza. La medida de adecuación muestral de Kaiser-Meyer-Olkin supone un valor de 0,738 , mientras que la prueba de esfericidad de Barlett ha obtenido un nivel de significación de 0,00 . Los factores obtenidos son los siguientes:

- Factor 1: Conocimiento de las situaciones de riesgo vividas por los hijos en Internet. Este factor está compuesto por las variables que recogen información sobre el grado de conocimiento que tienen los padres sobre riesgos experimentados por los hijos en la red como haber recibido o enviado mensajes desagradables o hirientes; haber tenido experiencias desagradables; amenazas, haber tenido acceso a imágenes sexuales o violentes o haberse citado con personas que ha conocido a través de la red. 
- Factor 2: Percepción de la brecha digital entre padres e hijos. Este factor incluye tres variables sobre el grado de inmersión digital de los hijos; limitaciones en la mediación por desconocimiento frente al hijo y sobre el intercambio de roles cuando es el hijo el que enseña a los padres a hacer uso de las TIC.

- Factor 3: Percepción sobre la necesidad de TIC en la sociedad. Este factor incluye tres variables sobre el grado de necesidad que los padres atribuyen a las tecnologías para las relaciones sociales, la educación y el mundo laboral.

- Factor 4: Preocupación por la capacidad de mediar. Este factor incluye dos variables sobre la necesidad de apoyo de profesores o expertos para ejercer la mediación y sobre la falta de capacidad para enseñar y proteger a los hijos en el contexto digital.

- Factor 5: Responsabilidad de los padres. Este factor incluye variables sobre la responsabilidad que tienen los padres de proteger a los hijos y de conocer los riesgos que viven en la red.

- Respecto a las preguntas con 4 posibles multirespuestas, se ha conseguido reducir 24 variables a 6 factores, que consiguen explicar el 61,5\% de la varianza con una medida de adecuación muestral de Kaiser-Meyer-Olkin que supone un valor de 0,816; mientras que la prueba de esfericidad de Barlett presenta un nivel de significación de 0,00 . Los factores identificados son:

- Factor 6: Preocupación por los riesgos de Internet. Este factor incluye 7 variables sobre situaciones que los hijos pueden vivir en internet que preocupan a los padres como el hecho de que busquen amigos en Internet; añadan a sus redes a desconocidos; finjan ser quienes no son; envíen información personal a desconocidos; sufran acoso o reciban contenidos sexuales o violentos.

- Factor 7: Conocimiento, uso compartido y control invasivo. Este factor incluye 5 variables sobre conocimiento a partir del uso compartido de internet, porque los padres se sientan con los hijos a navegar por la red y control invasivo porque los padres optan por comprobar lo que los hijos han hecho en la red y por revisar su correo además de recomendar sitios de internet y enseñarles herramientas o servicios.

- Factor 8: Conocimiento, presencia y control mediante la orientación y el diálogo. Incluye variables sobre el conocimiento que tienen los padres del uso y consumo que hacen los hijos de internet y sobre el diálogo sobre los riesgos que se puede establecer entre padres e hijos.

- Factor 9: Influencia de la televisión. Incluye tres variables sobre la percepción de la influencia de la televisión en los hijos en la forma de vestir, el lenguaje y el comportamiento.

- Factor 10: Control mediante uso compartido de las redes del padres. Incluye variables sobre la participación de los padres en las redes sociales de sus hijos y el tiempo de consumo del hijo.

- Factor 11: Equipamiento y tiempo de consumo. Incluye variables sobre la frecuencia con la el hijo navega por internet y si considera que su hogar está bien equipado. Por último, respecto a las preguntas con 5 posibles multirrespuestas, cabe destacar que se ha reducido 5 variables a 2 factores que consiguen explicar el 52,4\% de la varianza, con una medida de Kaiser-Meyer-Olkin de 0,589 y 0,00 de significación para la prueba de esfericidad de Barlett. Los dos factores identificados son: 
- Factor 12: Orientación y diálogo en el uso y consumo de televisión y presencia mientras navega. Agrupa variables sobre si los padres orientan sobre el consumo de televisión y sobre si hacen comentarios o conversan sobre los contenidos televisivos e incluye otra variable sobre la presencia de los padres mientras los hijos navegan.

- Factor 13: Percepción del tiempo dedicado a Internet. Agrupa variables sobre el tiempo que los padres pasan en Internet y sobre la valoración del tiempo que pasan los hijos en Internet.

A partir de los factores obtenidos, se ha procedido a establecer la tipología de estilos de mediación. La interpretación de los datos se ha hecho en torno a dos ejes: representación / estrategias de control. Para el análisis del primer eje se han tenido en cuenta los factores que tienen que ver con la percepción y preocupación sobre riesgos; conocimiento de los riesgos experimentados por los hijos; la percepción de la brecha digital y responsabilidad mediadora; conocimiento del uso y consumo e importancia atribuida a las TIC para el desarrollo de sus hijos. Para el análisis del segundo eje se han tenido en cuenta los factores que tienen que ver con estrategias de control, diálogo, uso compartido y orientación.

\subsection{Análisis y resultados}

El análisis cluster arroja 4 conglomerados diferenciados. La interpretación de los resultados nos permite definir cuatro modelos de familia que hemos denominado: preocupados ausentes, preocupados orientadores, despreocupados permisivos y despreocupados controladores.

- Preocupados ausentes: Es el grupo en el que existe una mayor percepción de la brecha digital. Son conscientes de la superioridad de sus hijos en el conocimiento de internet. Saben que sus hijos están más familiarizados que ellos en el uso de las TIC y esta situación les limita. En ocasiones es el hijo el que enseña a los padres a hacer uso de Internet. Este conjunto de padres tiene un alto conocimiento de las situaciones de riesgo vividas por los hijos; les preocupa que busquen amigos por la red, que añadan en sus listas de contacto a desconocidos, que finjan ser personas que no son o que sufran acoso o reciban contenidos eróticos. Esta puede que sea la razón por la que tienen una alta preocupación por su capacidad de mediar en el uso y consumo que hacen los hijos de las nuevas tecnologías. Consideran que los padres tienen responsabilidad relativa en el uso y consumo que los hijos hacen de las redes. Es un grupo de padres que considera que las TIC no son excesivamente necesarias para las relaciones sociales, el mundo laboral o la educación. Sí le atribuyen algo de influencia a la televisión en la forma de vestir, hablar o el comportamiento de los hijos. Son familias en las que el único control se hace poniendo límite al tiempo que los hijos pasan en Internet y en algunos casos participando en las redes sociales en las que están sus hijos. Son hogares en los que menos orientación y uso compartido se produce en relación a Internet y donde hay poco conocimiento del uso real que los hijos hacen de Internet y, por tanto, poco diálogo. Esta situación se repite en relación a la televisión. 
- Preocupados orientadores: Hay un segundo grupo de padres que presenta un alto grado de participación en el uso y consumo de Internet por parte de los hijos a través del conocimiento de lo que hacen los hijos en Internet, de la orientación, el diálogo y la presencia mientras hacen uso de las redes. En cambio no recurren a medidas de control sobre la limitación del tiempo y tampoco tratan de controlar estando presentes en las redes sociales en los que están los hijos. Son padres que tienen un alto grado de conocimiento de los riesgos que han vivido sus hijos en Internet y son los padres a los que más les preocupa esta cuestión; la posibilidad de que sus hijos experimenten riesgos en la red: buscar amigos en la red, añadir a desconocidos a sus listas de contacto, que se difundan imágenes hirientes sobre ellos, que reciba contenidos sexuales. Se trata del grupo de familia que más poder de influencia atribuye a la televisión sobre formas de vestir, comportamientos o el lenguaje y consideran que las TIC son necesarias para las relaciones sociales, el mundo laboral y la educación. Son los padres que más responsables se sienten de proteger a sus hijos de los peligros de la red y al mismo tiempo no le dan mucha importancia a la brecha porque es posible que no consideren que sus hijos sepan mucho más que ellos y, por tanto, no se sienten limitados en exceso a la hora de ejercer algún tipo de mediación.

- Despreocupados y permisivos: Hay un tercer grupo de padres que se caracterizan por no darle importancia al tipo de relación que sus hijos establecen con los medios y por tener un escaso conocimiento de lo que los hijos hacen en Internet. En primer lugar porque no consideran que el uso y consumo de TIC tenga influencias en los hijos sobre su forma de comportarse, de vestir o sobre su lenguaje y tampoco consideran que Internet sea importante para sus relaciones sociales, ni para el mundo laboral, ni para la educación. En este escenario, nos llama la atención que a lo que menos importancia le den sea a la orientación y al diálogo sobre Internet ni a acompañar a los hijos mientras navegan por la red; no participan en las redes sociales en las que están sus hijos. Tampoco establecen medidas de control sobre el tiempo que pasan los hijos haciendo uso de las TIC. Son padres que tienen escaso conocimiento sobre los riesgos que han experimentado los hijos en la red y tampoco es un asunto que les preocupe. No se sienten responsables de educar a los hijos en estas cuestiones ni perciben la brecha digital padres e hijos como un problema, ni es un asunto que les preocupe excesivamente.

- Despreocupados controladores: Se trata del grupo de padres que mayor importancia le da a las TIC para las relaciones sociales de los hijos, el mundo laboral y la educación. Muy por encima, de la influencia atribuida a la televisión. Son padres conscientes de que existe brecha digital pero no les preocupa en exceso su capacidad de mediar porque tampoco se sienten responsables de educar a los hijos en el uso y consumo de medios; responsabilidad que seguramente hagan recaer en otros entes con responsabilidad en la educación de los hijos como es el colegio. Son padres que conocen lo que los hijos hacen en internet y establecen medidas de control invasivas que consisten fundamentalmente en seguir el rastro de los hijos en internet para ver qué páginas han visitado y les vigilan el correo electrónico. El control directo también lo hacen mediante la recomendación de sitios en Internet y 
enseñándoles a usar determinadas herramientas y servicios. A lo que más importancia atribuyen en cuanto a estrategias de control es limitar el tiempo de consumo y estar presentes en las redes en las que participan los hijos para estar al tanto de lo que publican.

\section{Discusión y conclusiones}

Podemos trasladar al nuevo escenario algunas de las conclusiones de investigadores precedentes en contextos televisivos como la importancia del conocimiento que tengan los padres sobre el medio y sobre la experiencia de los hijos en el medio para generar una percepción que implique participación en el uso y consumo de los menores de los servicios digitales. Se evidencia la escasa formación de los padres para poder ejercer la mediación en un contexto todavía más complejo como es el digital.

Aunque en este artículo no difundimos resultados sobre el peso de cada estilo de mediación en la población, se percibe cierto solipsismo generalizado de los menores en uso de internet en sus hogares. Pero la contradicción que se percibe es que frente a la autonomía de los menores en el uso de los servicios digitales existe una mayor presión sobre los padres para controlar y regular ese uso. Presión que al mismo tiempo no va acompañada de una correcta formación de padres que les permite intervenir para favorecer una inclusión digital de los hijos satisfactoria. Surge la hipótesis de que es probable que la brecha digital en la actualidad no tenga tanto que ver con la divergencia en cuanto a competencia digital en su dimensión más técnica, sino en cuanto a los distintos usos sociales que hacen padres e hijos de internet que facilitan el encuentro ni una experiencia mediática común o compartida. ¿Qué prácticas se deben poner en marcha para informar y formar a los padres para que puedan responder a la llamada de participar en la educación mediática de los hijos? En este sentido, se ha planteado como objeto de estudio en una investigación ya iniciada, la propuesta de plantear la idea de la comunidad intergeneracional de aprendizaje como vía para que padres e hijos encuentren espacios de comunicación también en internet que les acerquen y generar en estos espacios nuevas estrategias de alfabetización digital que vayan más allá de las clásicas normas sobre tiempos, prohibiciones sobre servicios específico, etc.

Los resultados coinciden con estudios precedentes en la idea de que la necesidad de la alfabetización digital ha calado en el imaginario legislativo, académico pero todavía no ha calado en la sociedad. Esta investigación, en línea con la tradición culturalista de los Estudios de Recepción, incide en la importancia de la alfabetización digital de padres si se quiere afrontar el problema de la educomunicación de menores de forma efectiva. Consideramos que es necesaria la participación de los padres en la educación mediática de los hijos para garantizar una formación íntegra y que la inclusión en la cultura digital sea conforme a su dignidad de seres humanos y que contribuyan con su participación en los nuevos medios a hacer la sociedad más humana. Para ello es necesario desplegar estrategias para la concienciación y la formación de las familias. Necesidad de estudios etnográficos, sobre experiencia mediática, e incluir variables familiares. 


\section{Referencias bibliográficas}

Aguaded Gómez, José Luis (2010): “La educación para la comunicación. Nueva alfabetización para un mundo global”, en Del Moral, María Ester (coord.): Televisión: desarrollo de la creatividad e infancia. Barcelona, Octaedro, pp. 55-70.

Aguaded Gómez, José Luis (2011): "La educación mediática, un movimiento internacional imparable. La ONU, Europa y España apuestan por la educomunicación”. Comunicar, 37, (XIX), 7.

Airebe, Ana; Orozco, Guillermo; y Medrano, Concepción (2014): “Family context, televisión and perceived values. A cross-cultural studywithadolescents". Comunicación y Sociedad, 27 (2), 79-99.

Area, Manuel (2012): “Alfabetización en la sociedad digital”, en Area, Manuel; Gutiérrez, Alfonso; y Vidal, Fernando: Alfabetización digital y competencias informacionales. Madrid, Fundación Encuentro y Fundación Telefónica, pp. 3-43.

Bringué, Xabier y Sádaba, Charo (2009): La Generación Interactiva en España. Niños y Adolescentes ante las Pantallas. Barcelona, Colección Fundación Telefónica, Ariel.

Bronfenbrenner, Uriel (1987): La ecología del desarrollo humano. Barcelona, Ediciones Paidós.

Ferres Prats, Joan; Aguaded, José Luis; y García Matilla, Agustín (2012): “La competencia mediática de la ciudadanía española: dificultades y retos". Icono 14, 10 (3), 23-42.

Fundación Telefónica (2015): La Sociedad de la Información en España 2014. Madrid, Ariel.

Garmendia, Maialen; Casado, Miguel Ángel; Martínez, Gemma; y Garitaonandia, Carmelo (2013): "Las madres y padres, los menores e internet. Estrategias para la mediación parental en España”. Doxa, 17, 99-117.

Garmendia, Maialen; Garitaonandia, Carmelo; Martínez, Gemma; y Casado, Miguel Ángel (2011): Riesgos y seguridad en internet: Los menores españoles en el contexto europeo. Universidad del País Vasco/Euskal Herriko Unibertsitatea. Bilbao, EU Kids Online.

Gutiérrez, Alfonso y Tyner, Kathleen (2012): “Educación para los medios, alfabetización mediática y competencia digital". Comunicar, 38 (19), 31-39.

Haddon, Leslie (2015): "Children's Critical Evaluation of Parental Mediation", en Cyberpsychology, 2, en:

http://cyberpsychology.eu/view.php?cisloclanku=2015051402yarticle=2. [Consulta: $1 \mathrm{de}$ marzo de 2015]

Haddon, Leslie (2012): "Parental mediation of internet use: evaluating family relationships", en: Loos, Eugene; Haddon, Leslie; y Mante-Meijer, Enid (Eds.): Generational Use of New Media. UK, Ashgate, Farnham, pp. 13-30.

Hall, Stuart (2004): "Codificación y descodificación en el discurso televisivo". CIC: Cuadernos de información y comunicación, 9, 215-236.

Islas Carmona, José Octavio (2009): "El prosumidor. El actor comunicativo de la sociedad de la ubicuidad". Palabra Clave, no 11 (1), 29-39.

Kendal, Alex y Mcdougall, Julián (2012): “Alfabetización mediática crítica en la postmodernidad". Comunicar, 38, 21-29.

Livingstone, Sonia \& Bober, Magdalena (2006): "Regulating the Internet al home: Contrasting the perspectives of children and parents", en Buckingham, David \& Willett, Rebekah (Eds.): Digital generations. Mahwah, NJ, Laurence Erlbaum Associates, Inc, pp. 93-113.

Livinsgtone, Sonia \& Helsper, Ellen (2008): "Parental mediation and children's Internet use". Journal of Broadcastingyelectronic media, $\mathrm{n}^{\circ} 52$ (4), 581-599. 
Lull, James (1997): Medios, comunicación, cultura. Aproximación global. Buenos Aires, Amorrortu.

Marciales Vivas, Patricia y Cabra Torres, Fabiola (2011): "Internet y pánico moral: revisión de la investigación sobre la interacción de niños y jóvenes con los nuevos medios". Univ. Psychol, 10 (3), 855-865.

Marta Lazo, Carmen y Grandío, Mar (2013): “Análisis de la competencia audiovisual de la ciudadanía española en la dimensión de recepción y audiencia”. Communication y Society, 26 (2), 114-130.

Martín Barbero, José Luis (1987): De los medios a las mediaciones. Méjico, Gustavo Gili.

Morley, David (1986): Family televisión: Cultural power and domestic leisure. Buenos Aires, Amorrortu.

Morley, David (1996): Televisión, audiencias y estudios culturales. Buenos Aires, Amorrortu.

Orozco, Guillermo (1996): Televisión y audiencias. Un enfoque cualitativo. Madrid, Ediciones de la Torre.

Orozco, Guillermo; Navarro, Eva; y García Matilla, Agustín (2012): "Desafíos educativos en tiempos de auto-comunicación masiva: la interlocución de las audiencias". Comunicar, $38,67-74$.

Pérez Sánchez, Ramón (2013): Infancia, socialización y TIC, en Informe PROSIC 2013. Hacia la Sociedad de la Información y el Conocimiento. PROSIC, Costa Rica, pp. 343368.

Pérez Tornero, José Manuel y Tapio, Varis (2012): Alfabetización mediática y nuevo humanismo. Barcelona, Editorial UOC.

Pérez Tornero, José Manuel (2015): "La emergencia de la alfabetización mediática e informacional Un nuevo paradigma para las políticas públicas y la alfabetización”. Telos Cuadernos de Comunicación e Innovación, 100, 99-102.

Sandoval, Yamile y Aguaded, Ignacio (2012): "Nuevas audiencias, nuevas responsabilidades". ICONO 14, 10 (3), 8-22.

Staksrud, Elisabeth \& Livingstone, Sonia (2009): “Children and online risk: powerless victims or resourceful participants?”. Information, communication and society, 12 (3), 364-387.

Valkenburg, Patt; Krcmar, Marina; Peeters, Allerd; and Marseille, Nies (1999): "Developing a scale to assess three styles of television mediation: instructive mediation, restrictive mediation, and social coviewing”. Journal of Broadcasting y Electronic Media, 43 (1), 52-67.

Van der Voort, Tom H.A.; Nikken, Peter; and Van Lil, J.E. (1992): "Determinations of parental guidance of children's television viewing: A Dutch replication study". Journal of Broadcasting y Electronic Media, 36, 61-74.

Teresa Torrecillas-Lacave es Profesora Contratada Doctora del Departamento de Periodismo de la Universidad CEU San Pablo de Madrid.

María Encina Morales es Profesora Colaboradora Doctora del Departamento de Economía Aplicada de la Universidad CEU San Pablo de Madrid.

Tamara Vázquez-Barrio es Profesora Contratada Doctora del Departamento de Periodismo de la Universidad CEU San Pablo de Madrid. 